\title{
New air pollution evaluation index based on AQI
}

\author{
Shan Gao ${ }^{1, a)}$ \\ ${ }^{1}$ North China Electric Power University, Baoding 071000,China. \\ a)1786831655@qq.com
}

\begin{abstract}
Our goal is a model that can evaluate the grade of air quality index and a model can calculate the air quality index in a synthetic way.The air quality index considers the effect of six pollutants(PM2.5,PM10,SO2,NO2,CO,O3).We can find O3 has so little influence on AQI that we can ignore the effect of O3.We standardize all data in order to simplify the analysis procedure.We carefully examine the relationship between AQI and each pollutant and work out the weigh of each pollutant in AQI.Then we integrate multiple effects to derive a synthetic evaluation which called Basic M_AQI Model.Basic M_AQI Model can be divided into six levels including excellent,moderate,slightly polluted,moderately polluted,heavily polluted and seriously polluted.Finally,we compile the list of pros and cons of the model.We give an objective assessment to the model and put forward recommendations for improving the model.
\end{abstract}

Keywords: air quality index, standardized treatment, trend similarity, comprehensive evaluation

\section{INTRODUCTION}

The effect of air pollution index is based on ambient air quality standards and the various pollutants on human health and ecological environment, and several air pollutant concentrations in routine monitoring is simplified as single concept refers to the numerical form, it will be graded the degree of air pollution and air quality that is suitable for representing the city air quality status and trends. ${ }^{[1]} \mathrm{AQI}$ index only represents the degree of air pollution.Air pollutants include PM2.5,PM10,SO2,NO2,CO,O3 ${ }^{[2]}$.We tackle a main problem:Build evaluate model that is named after M_AQI.M_AQI can evaluate the air quality and divide air quality into six grades.we find the degree of fitting between AQI and air pollutants.Then,add the effect of each to get M_AQI.

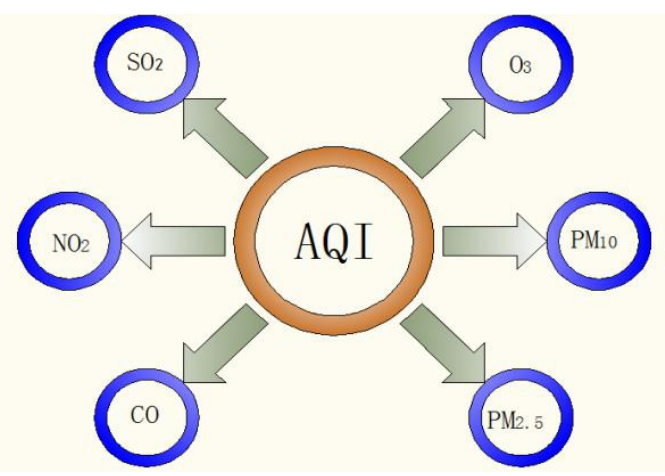

FIGURE1:Schematic diagram of AQI

Known AQI index considers only primary pollutants,so we have improved on this basis considering other indicators to make the evaluation system more reasonable.

\section{ASSUMPTIONS}

To simplify the problem,we make the following basic assumptions,each of which is properly justified.

1.Six indexes of air pollution reach peak or trough almost at the same time.This means that the situation of only one index incredibly high or low can not happen. Figure 1 demonstrates what we suppose above.

2.The given database is representative of the all the condition. One hundred and fifty days include six grades.So,database can reflect all condition.

3.The trend of AQI in a short period of time will not have a large fluctuation.In fact,air quality indexes have a lot relationship with time especially a few days ago.So,the trend of AQI will not change with no reason in a short of time.

4.We suppose the extremely pointed area is abnormal.This assumption is reasonable and can simplify the question. 


\section{NOTATIONS}

All the variables and constants used in this paper are listed in Table $\mathbf{1 .}$

TABLE1:symbol

\begin{tabular}{ccc}
\hline Symbol & Definition & Units \\
\hline$A_{j}^{i}$ & the initial index of air pollutant $\mathrm{j}$ at the $\mathrm{i}$-day & dimensionless \\
$A_{j}^{\text {min }}$ & the minimal initial index of air pollutant $\mathrm{j}$ & dimensionless \\
$A_{j}^{\max }$ & the maximal initial index of air pollutant $\mathrm{j}$ & dimensionless \\
$E_{j}^{i}$ & the standardized index of air pollutant $\mathrm{j}$ at the $\mathrm{i}$-day. & dimensionless \\
$Q_{i}$ & the initial index of AQI & dimensionless \\
$R_{i}$ & the standardized index of AQI & dimensionless \\
$\beta_{j}$ & the fit indicator of air pollution & dimensionless \\
$n$ & the number of day of given date & day \\
$M_{-} A Q I_{i}$ & the index of air pollution at the i-day & dimensionless \\
$\chi_{g}$ & the average M_AQI of g grade & dimensionless \\
$g_{n}$ & the number of g grade & number \\
$\alpha_{g}$ & a new watershed of g grade and $(\mathrm{g}+1)$ grade & dimensionless \\
$g_{\text {nabove }}$ & the number of g grade above $\chi_{g}$ & number \\
$(g+1)_{\text {nabove }}$ & the number of (g+1) grade above $\chi(g+1)$ & number \\
$A_{j}^{\text {min }^{\text {min }}}$ & the minimal index of air pollutant $\mathrm{j}$ in history & dimensionless \\
$A_{j}^{0^{\text {max }}}$ & the maximal initial index of air pollutant $\mathrm{j}$ in history & dimensionless \\
$E_{j}^{0^{i}}$ & the standardized index of air pollutant $\mathrm{j}$ at the $\mathrm{i}$-day universality & dimensionless \\
$R_{0 i}$ & the standardized index of AQI universality & dimensionless \\
\hline
\end{tabular}

\section{MODEL ESTABLISHMENT}

We will start with the idea of the basic model.Basic M_AQI model can deal with the given data.Then we present and explain algorithm.Finally,we simply evaluate this basic model.

\section{Data Standardization}

Before the data analysis, we usually need to standardize the data (normalization), using standardized data for data analysis. Data standardization is the index of statistical data. Data standardization includes two aspects: data processing and non dimensional processing. Data processing is mainly to solve the problem with the chemotactic properties of different data, comprehensive results correctly reflect the different forces directly add not to different indicators, must first consider changing the inverse index data in nature, so that all indexes of force on the assessment scheme with chemotaxis, plus the total in order to obtain the correct results. Non dimensional data processing mainly solves the comparability of data. ${ }^{[3]} \mathrm{After}$ the data is standardized,all the data are transformed into dimensionless indicators.We directly analyze and process standardized data.

$$
\begin{aligned}
E_{j}^{i} & =\frac{A_{j}^{i}-A_{j}^{\min }}{A_{j}^{\max }-A_{j}^{\min }} \\
R_{i} & =\frac{Q_{i}-Q_{\min }}{Q_{\max }-Q_{\min }}
\end{aligned}
$$

$A_{j}^{i} \quad$ is the initial index of air pollutant $j$ at the $i$-day.

$A^{\text {min }}{ }_{j}$ is the minimal initial index of air pollutant $j$.

$A^{\max }{ }_{j}$ is the maximal initial index of air pollutant $j$.

$E_{j}^{i}$ is the standardized index of air pollutant $j$ at the $i$-day.

$Q_{i} \quad$ is the initial index of AQI.

$R_{i} \quad$ is the standardized index of AQI.

The curve of standardized data is in the following figure. 


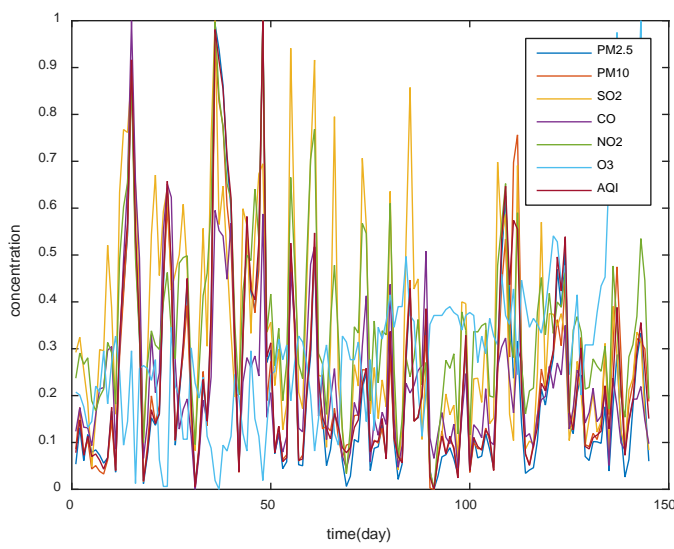

FIGURE2.after standardized treatment

We find that there is a big difference between the light blue curve and other curves in the image.So we eliminate the ozone parameters, to make the following curves like following figure.

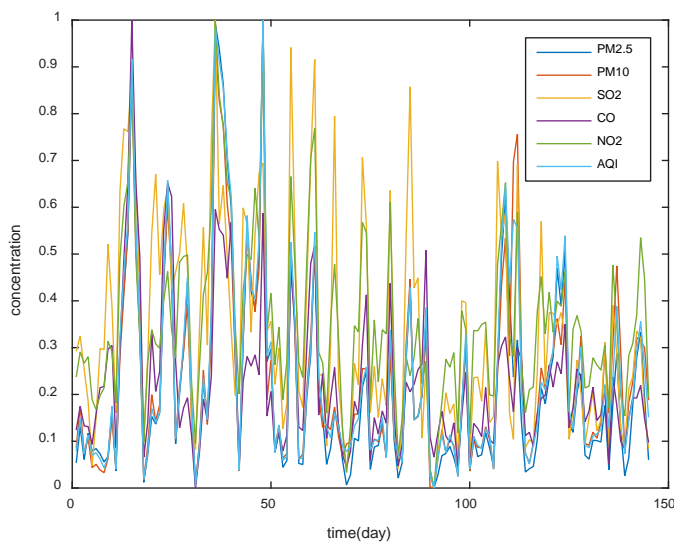

FIGURE3.after standardized treatment(except O3)

From this image, we find that all the curves have high similarity, so we choose these parameters as the main evaluation index.

\section{The Fit Indicator}

Different air pollutants have different contributions to AQI. If we know the fit of each standardized pollutant index with standardized index of AQI,we can work out the weigh of each pollutant in AQI.

The following is the algorithm:

$$
\beta_{j}=\frac{1}{\sum_{i=0}^{i=n}\left(R_{i}-E_{j}^{i}\right)}
$$

$\beta_{j}$ is the fit indicator of air pollutant $j$.

$n$ is the number of days in given data.

The analysis of $\beta_{j}$ might shed light on the fit indicator of air pollutant $j$.We describe the fit indicator by the gap between the standardized index of AQI and standardized index of air pollutant $j$ at the i-day.If the value of $\beta_{j}$ is bigger,the similarity of two curve is better. $\beta_{O 3}$ is so low that $\mathrm{O} 3$ has little influence on AQI.In order to simplify the question,we can ignore the effect of $\mathrm{O} 3$.

TABLE2.the fit indicator of air pollution

\begin{tabular}{cccccc}
\hline$\beta_{j}$ & $\beta_{S O 2}$ & $\beta_{N O 2}$ & $\beta_{C O}$ & $\beta_{P M 2.5}$ & $\beta_{P M 10}$ \\
\hline weight & 0.0467 & 0.0517 & 0.0779 & 0.2803 & 0.2509 \\
\hline
\end{tabular}

\section{The Value of M AQI}

According to the fit indicator of air pollutant $\mathrm{j}$,we can distribute the weigh of pollutant $\mathrm{j}$ in our system.So we derive the contribution of pollutant $\mathrm{j}$ at $\mathrm{i}$-day in $\mathrm{M} \_\mathrm{AQI}$ is $\beta_{j} \times E_{j}^{i}$

Then we can derive M_AQI of i-day. 


$$
M_{-} A Q I_{i}=\sum_{j=1}^{5}\left(\beta_{j} \times E_{j}^{i}\right)
$$

M_AQI is the index of air pollution which concludes five air pollutants .M_AQI can give a synthetic evaluation.Following figure can show the value of M_AQI.Different colors represent the classification of the original standard,we show them in the following table.

TABLE3.air quality of different grades (original standard)

\begin{tabular}{ccccccc}
\hline color & red & blue & green & black & sky blue & pink \\
\hline \multirow{2}{*}{ standard grade } & \multirow{2}{*}{ excellent } & \multirow{2}{*}{ moderate } & \multirow{2}{*}{ slightly polluted } & \multirow{2}{*}{ moderately polluted } & heavily polluted & seriously polluted \\
\hline
\end{tabular}

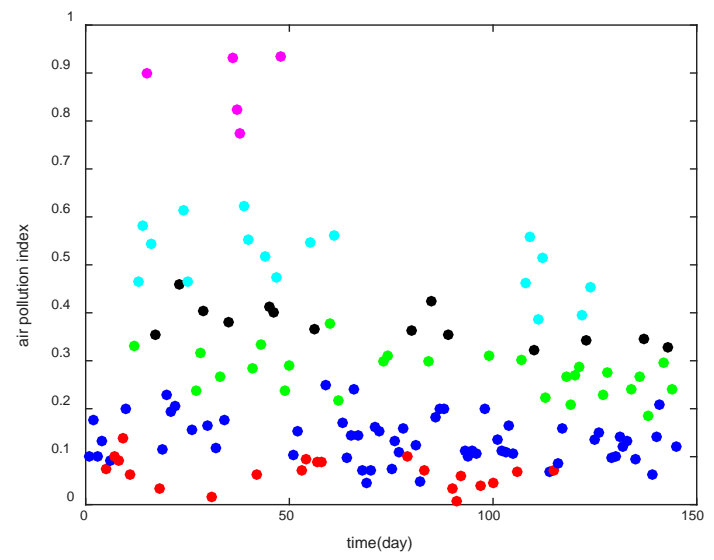

FIGURE4.scatter plot of air pollution index

We found that the air quality in the original standard has a certain regularity in the new evaluation system,so we try to use the new evaluation index to classify the air quality. We give different levels of classification by the following methods.

In order to give a clear degree of air pollution,we divided the M_AQI according to the numerical value.M_AQI also can be divided into six levels including excellent,moderate,slightly polluted,moderately polluted,heavily polluted,seriously polluted.Figure 3 indicates that grades of M_AQI is different from grades of AQI.We can calculate the average M_AQI of each grade.

In order to calculate the boundary of different grades, we designed the following calculation methods

$$
\begin{gathered}
\chi_{g}=\frac{\sum_{a=1}^{g_{n}} M_{-} A Q I}{g_{n}} \\
\alpha_{g}=\chi_{g}+\left(\chi_{(g+1)}-\chi_{g}\right) \times \frac{g_{\text {nabove }}}{\left((g+1)_{\text {nbelow }}+g_{\text {nabove }}\right)}
\end{gathered}
$$

$\chi_{g}$ is the average of M_AQI of g grade.

$g_{n}$ is the number of g grade.

$\alpha_{g}$ is a new watershed of $g$ grade and $(g+1)$ grade.

$g_{\text {nabove }}$ is the number of $g$ grade above $\chi_{g}$.

$(g+1)_{\text {nbelow }}$ is the number of $(g+1)$ grade below $\chi(g+1)$.

\begin{tabular}{|c|c|c|c|c|c|}
\hline$\alpha_{\text {excellent }}$ & $\alpha_{\text {moderate }}$ & $\alpha_{\text {slightly }}$ & $\alpha_{\text {moderately }}$ & $\alpha_{\text {heavily }}$ & $\alpha_{\text {excellent }}$ \\
\hline $0 \sim 0.1007$ & $0.1007 \sim 0.2019$ & $0.2019 \sim 0.3222$ & $0.3222 \sim 0.4454$ & $0.4454 \sim 0.7274$ & $0.7274 \sim 1$ \\
\hline
\end{tabular}

The ranges obtained by this method are shown in the following table.

TABLE4.Scope of each grade

\section{CONCLUSION}

The model we established in the original AQI model has been improved to enhance the impact of various pollutants on the evaluation index, taking into account the impact of different pollutants on the evaluation index. In addition, a new method for calculating the air quality evaluation index is given.Our model is based on the data from a certain area,so compared with the original evaluation method, the evaluation model of our model is more consistent with the local 
reference value.The main content of this model is to put forward an improved method in the original AQI evaluation system,the most important thing in the article is its calculation method.

\section{ACKNOWLEDGMENTS}

First and foremost, I would like to show my deepest gratitude to my teacher, Mr.Weiliang Liu, a respectable, responsible and resourceful scholar, who has provided me with valuable guidance in every stage of the writing of this thesis. Without his enlightening instruction, impressive kindness and patience, I could not have completed my thesis. His keen and vigorous academic observation enlightens me not only in this thesis but also in my future study. I would also like to thank all my teachers who have helped me to develop the fundamental and essential academic competence.

\section{REFERENCES}

[1] Ying YUAN,Ming LIU, Differences of Air Quality Index (AQI) and Air Pollution Index (API),Guangzhou Chemical Industry Vol. 42 No. 12. Jun. 2014

[2] http://kjs.mep.gov.cn/hjbhbz/bzwb/dqhjbh/jcgfffbz/201203/W020120410332725219541.pdf

[3] Lixing Jiao . On methods of Standardization Mangement of Index, Journal of UEST of China,vol. 26 supp. Apr.1997 